\title{
Metal-Enhanced Fluorescence-Based RNA Sensing
}

\author{
Kadir Aslan, ${ }^{\dagger}$ Jun Huang, ${ }^{\ddagger}$ Gerald M. Wilson, ${ }^{\ddagger}$ and Chris D. Geddes ${ }^{\star}, \uparrow, \S$ \\ Institute of Fluorescence, Laboratory for Advanced Medical Plasmonics, Medical Biotechnology Center, University \\ of Maryland Biotechnology Institute, and Department of Biochemistry and Molecular Biology and Center for \\ Fluorescence Spectroscopy, University of Maryland School of Medicine, Baltimore, Maryland 21201
}

Received January 6, 2006; E-mail: geddes@umbi.umd.edu

Quantitation of specific RNA molecules from biological samples is an essential tool for the study of regulated gene expression ${ }^{1}$ and is routinely employed in studies of gene transcription, ${ }^{2}$ RNA stability, ${ }^{3}$ RNA transport, and a host of other biological processes. ${ }^{4}$ In addition, RNA detection and quantitation also present an appealing strategy for rapidly identifying unknown biological agents (bacterial, viral, etc.). ${ }^{5,6}$ Furthermore, they are of great utility for gene expression profiling in clinical settings, where the expression of a subset of genes within tissue (i.e., biopsy) or blood samples may be rapidly measured, revealing diagnostic information to direct patient-specific therapeutic strategies. ${ }^{2,7}$

All current techniques for quantifying specific RNAs exploit base pair complementarity between a target RNA and one or more nucleic acid probes, either in the form of extended DNA or RNA sequences (Northern blots, ${ }^{1}$ RNase protection assays ${ }^{8,9}$ [RPAs]) or short oligonucleotides (reverse transcription-PCR [RT-PCR], ${ }^{10}$ RNA capture assays ${ }^{11}$ ). This principle allows for extremely precise target recognition, yet current methods of probe:target hybrid detection face a number of technological restrictions. In particular, the utility of RNA sensing in microbial detection and/or clinical gene expression profiling may be hindered by two principal constraints, namely, sensitivity and rapidity. ${ }^{12}$

RNA capture assays offer a simple and rapid approach to RNA quantitation. Target RNAs are selected based on complementarity to an oligonucleotide probe which is attached to a solid surface or matrix, then detected by annealing a radio- or chemically labeled probe at a distinct site on the target RNA. ${ }^{11}$ At present, however, these assays are subject to the same sensitivity limitations as those described for Northern blots and RPAs, namely, that detection relies on the activity of radiolabels, the sensitivity of conjugated fluorophores, or the use of bright secondary chemiluminescent assays. These conditions make RNA capture assays currently useful only for abundant RNA species, thus limiting their general utility as a biosensor platform. ${ }^{10}$

Here we present the proof-of-principle of a new RNA sensing platform technology based on Metal-Enhanced Fluorescence (MEF), where the detected fluorescence emission is significantly amplified and we were able to trace the varying amount of RNA on the silver island films ( $\mathrm{SiFs}$ ) quantitatively as compared to the undetectable emission on the non-silvered glass surface at the femtomole level detection of RNA. In this regard, the detection of RNA is accomplished by annealing a target RNA, tagged with a fluorophore, to an oligonucleotide anchor probe in a single step on a solid surface, where the fluorescence signal is intrinsically enhanced by silver nanoparticles. The enhancement of fluorescence is, in part, due to the localized excitation of the fluorophores when in close proximity to the silver nanoparticles and results in improved

\footnotetext{
$\dagger$ Institute of Fluorescence, Laboratory for Advanced Medical Plasmonics.

₹ Department of Biochemistry and Molecular Biology.

$\S$ Center for Fluorescence Spectroscopy.
}

Scheme 1. MEF-Based RNA Sensing Platform Technology

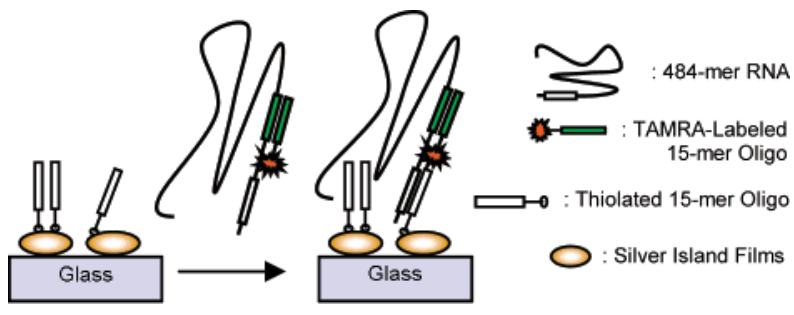

photostability of the fluorophores. ${ }^{13,14}$ When the metal (silver, aluminum, or gold) is a continuous $45 \mathrm{~nm}$ thick film, the spatially isotropic fluorescence emission can be converted into directional emission toward a detector, further improving the detectability. ${ }^{16}$

The RNA capture assay was constructed by covalently linking a thiolated oligo anchor probe (thiolated 15-mer oligo) onto SiFs via well-established self-assembled monolayer chemistry, ${ }^{17}$ as shown in Scheme 1. The thiolated oligo anchor probe is designed by introducing thiol groups into the 15 -base sequence from the $5^{\prime}$ end that specifically binds to silver nanoparticles with high affinity. A fluorescent probe (TAMRA-labeled 15-mer oligo) is annealed to a 484-mer RNA substrate containing the $\beta$-globin coding sequence (target RNA; see Figure S1, Supporting Information). The TAMRA-labeled oligo annealed to the RNA substrate was then hybridized with the thiolated oligo anchor probe on the surface of the SiFs. This procedure brings the fluorophore to a distance of approximately $4 \mathrm{~nm}$ from the surface of the SiFs where the fluorescence emission is expected to increase by MEF as described previously. ${ }^{13,14}$

The deposition of silver island films onto glass slides was performed as described previously. ${ }^{18}$ In a typical SiF preparation, a solution of sodium hydroxide and ammonium hydroxide is added to a continuously stirred solution of silver nitrate at room temperature. Subsequently, the mixture is cooled in an ice bath, silaneprep glass slides (Sigma) are inserted, and a solution of D-glucose is added. As the temperature is increased, the color of the mixture turns yellow-brown and the SiF-deposited slides are removed from the mixture, washed with water, and sonicated for a few seconds at room temperature. SiF-deposited glass slides were stored in deionized water until they were used. Fluorescence emission spectra of TAMRA-labeled oligo with RNA substrate hybridized to the thiolated oligo anchor probe on SiFs are shown in Figure 1, left. The emission intensity peak of TAMRA-labeled oligo that was annealed to RNA substrates ranging from 25 to $500 \mathrm{fmol}$ is clearly observed at $585 \mathrm{~nm}$ (Figure 1, left) and increased linearly as the amount of RNA substrate is increased (Figure 1, right). The fluorescence emission spectra of TAMRA shown in Figure 1, left (especially for the RNA substrates of $250 \mathrm{fmol}$ or higher) appear slightly broader than the spectrum of TAMRA-labeled oligo anchor probe measured from a solution on plain glass (see Figure S2, 

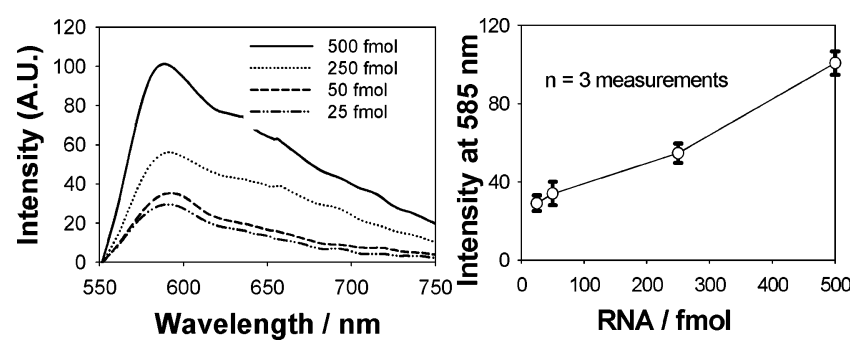

Figure 1. (Left) Fluorescence emission spectra (intensity: arbitrary units) of TAMRA-linked oligo annealed to the RNA substrate that was hybridized with the thiolated oligo anchor probe on the surface of the SiFs. (Right) Fluorescence emission intensity measured at $585 \mathrm{~nm}$ versus the amount of RNA used in the RNA capture assay (signal-to-noise, S/N > 20) for three separate measurements (background fluorescence is 1.5 at $517 \mathrm{~nm}$; see Figure S5).

Supporting Information) due to the background scattering from the SiF-coated glass slide.

The control experiments revealed that when the RNA sequence was changed (that is, control tRNA with random sequence is used in the RNA capture assay) the fluorescence emission from TAMRAlabeled oligo was not observed (see Figure S3, Supporting Information) since the control tRNA lacked the specific sequence that is required for the annealing of TAMRA-labeled RNA. In addition, when either of the other components of the RNA capture assay, thiolated oligo, or TAMRA-labeled oligo is omitted, almost no fluorescence emission was observed (see Figure S3, Supporting Information). Thus, the RNA capture assay is highly specific, and the contribution of the nonspecific interactions to the detected signal is minimal.

The lower detection limit (LDL) of the RNA capture assay described here was $25 \mathrm{fmol}$ of RNA ( $/ \mathrm{N}>20)$ and made possible by the amplification of fluorescence emission intensity based on our previously described phenomenon of metal-enhanced fluorescence. ${ }^{13,14}$ The amplification of fluorescence emission intensity is an inherent property of the silver nanoparticles deposited on the glass slides and thought to occur due to partial nonradiative energy transfer between the excited state of the fluorophore and the surface plasmons of the silver nanoparticles, as well as due to the spatially localized excitation of fluorophores created by the nanoparticles within close proximity. ${ }^{19}$

Although the LDL of the MEF-based RNA capture assay is 100200-fold less sensitive than the current RNA capture assays, ${ }^{11,22}$ the MEF-based RNA sensing method offers a considerably simpler, cheaper, and quicker alternative to RT-PCR since it does not require the amplification of the RNA target and can be performed relatively quickly. Given that the $\mathrm{S} / \mathrm{N}>3-4$ for fluorescencebased assays is considered acceptable, ${ }^{15}$ the actual lower detection limit of the MEF-based RNA capture assay is approximately $5 \mathrm{fmol}$.

The rapidity of the MEF-based RNA capture assays could be increased further with the help of low-power microwaves, as shown previously for the MEF-based protein and antibody assays that were completed within $20 \mathrm{~s}$, that is, microwave-accelerated metalenhanced fluorescence (MAMEF). ${ }^{18,20}$ Similar to RT-PCR, the
MEF-based RNA capture assays could potentially be multiplexed by simply using SiF-coated high-throughput screening (HTS) wells. $^{21}$ Ultimately, ultra-rapid MEF-based multiplexed RNA capture assays comparable to RT-PCR could be achieved by combining MAMEF technology with the use of SiF-coated HTS wells once the sensitivity of the MEF-based method is improved. In this regard, our laboratories recently reported MEF-based enhancements in excess of 3000-fold using fractal silver surfaces. ${ }^{14}$ Accordingly, the timing is propitious for the application of metalenhanced fluorescence to an RNA quantitation platform.

Acknowledgment. This work was supported by the NIH GM070929 and the National Center for Research Resources, RR008119. Partial salary support to C.D.G. from UMBI/MBC is also acknowledged.

Supporting Information Available: The experimental conditions for the preparation of silver island films, the preparation of the $\beta$-globin mRNA substrate, and the MEF-based RNA sensing assay are provided. This material is available free of charge via the Internet at http:// pubs.acs.org.

\section{References}

(1) Krumlauf, R. Mol. Biotechnol. 1994, 2, 227-242.

(2) Elkahloun, A. G.; Gaudet, J.; Robinson, G. S.; Sgroi, D. C. Cancer Biol. Ther. 2002, 1, 354-358.

(3) Wilson, G. M.; Deeley, R. G. Plasmid 1995, 33, 198-207.

(4) Kindler, S.; Wang, H.; Richter, D.; Tiedge, H. Annu. Rev. Cell Dev. Biol. 2005, 21, 223-245.

(5) van Doorn, L. J.; Kleter, B.; Voermans, J.; Maertens, G.; Brouwer, H.; Heijtink, R.; Quint, W. J. Med. Virol. 1994, 42, 22-28.

(6) Call, D. R.; Borucki, M. K.; Loge, F. J. J. Microbiol. Methods 2003, 53 , $235-243$.

(7) Ramaswamy, S.; Golub, T. R. J. Clin. Oncol. 2003, 20, 1932-1941.

(8) Haines, D. S.; Gillespie, D. H. Biotechniques 1992, 12, 736-741.

(9) Rosenau, C.; Kaboord, B.; Qoronfleh. M. W. Biotechniques 2002, 33, $1354-1358$

(10) Bustin, S. A. J. Mol. Endocrin. 2002, 29, 23-39.

(11) Tsai, S. P.; Wong, A.; Mai, E.; Chan, P.; Mausisa, G.; Vasser, M.; Jhurani, P.; Jakobsen, M. H.; Wong, W. L. T.; Stephan, J.-P. Nucleic Acids Res. 2003, 31, e 25

(12) The sensitivity of RNA detection becomes limiting when only minute quantities of biological material are available. This is also reflected in poor signal-to-noise when the RNA of interest is expressed at very low levels relative to the bulk RNA population (e.g., expression of oncogene mRNAs as a function of total cell RNA mass, low levels of viral mRNAs in a blood sample from a patient with a latent infection). The rapidity is highly desirable in an RNA sensing system, particularly in microbial screening, since early identification of pathogens provides better opportunities for containment, decontamination, and treatment.

(13) Aslan, K.; Gryczynski, I.; Malicka, J.; Matveeva, E.; Lakowicz, J. R.; Geddes, C. D. Curr. Opin. Biotechnol. 2005, 16, 55-62.

(14) Parfenov, A.; Gryczynski, I.; Malicka, J.; Geddes, C. D.; Lakowicz, J. R. J. Phys. Chem. B 2003, 107, 8829-8833.

(15) Lakowicz, J. R. Principles of Fluorescence Spectroscopy; Kluwer: New York, 1999.

(16) Malicka, J.; Gryczynski, I.; Gryczynski, Z.; Lakowicz J. R. J. Biomol. Screen. 2004, 9, 208-215.

(17) Sastry, M.; Mayya, K. S.; Bandyopadhyay, K. Colloids Surf. A 1997, 127, $221-228$.

(18) Aslan, K.; Geddes, C. D. Anal. Chem. 2005, 77, 8057-8067.

(19) Aslan, K.; Leonenko, Z.; Lakowicz, J. R.; Geddes, C. D. J. Fluoresc. 2005, 15, 643-654.

(20) Aslan, K.; Geddes, C. D. J. Fluoresc. 2006, DOI:10.1007/s10895-0050026-z.

(21) Aslan, K.; Holley, P.; Geddes, C. D. J. Immun. Methods, submitted.

(22) Xie, H.; Yu, Y. H.; Xie, F.; Lao, Y. Z.; Gao, Z. Anal. Chem. 2004, 76, $4023-4029$.

JA0601179 


\section{Supporting Information for the Manuscript:}

"Metal-Enhanced Fluorescence Based RNA Sensing” by Kadir Aslan, Jun Huang, Gerald M. Wilson and Chris D. Geddes.

\section{S1.0. Materials and Methods}

\section{S1.1. Materials}

Silver nitrate (99.9\%), sodium hydroxide (99.996\%), ammonium hydroxide (30\%), trisodium citrate, $D$-glucose and premium quality APS-coated glass slides $(75 \times 25 \mathrm{~mm})$ were obtained from Sigma-Aldrich. The sources for enzymes, RNA and DNA are given in section S1.2.3.

\section{S1.2. Methods}

\section{S1.2.1. Formation of Silver Island Films (SiFs) on APS-coated Glass Substrates}

In a typical $\mathrm{SiFs}$ preparation a solution of silver nitrate $(0.5 \mathrm{~g}$ in $60 \mathrm{ml}$ of deionized water) in a clean 100-ml glass beaker, equipped with a Teflon-coated stir bar, is prepared and placed on a Corning stirring/hot plate. While stirring at the quickest speed, $200 \mu \mathrm{L}$ of freshly prepared $5 \%(\mathrm{w} / \mathrm{v})$ sodium hydroxide solution are added. This results in the formation of dark brown precipitates of silver particles. Approximately $2 \mathrm{ml}$ of ammonium hydroxide is then added, drop by drop, to re-dissolve the precipitates. The clear solution is cooled to $5^{\circ} \mathrm{C}$ by placing the beaker in an ice bath, followed by soaking the APS-coated glass slides in the solution. While keeping the slides at $5^{\circ} \mathrm{C}$, a fresh solution of $D$-glucose $(0.72 \mathrm{~g}$ in $15 \mathrm{ml}$ of water) is added. Subsequently, the temperature of the mixture is then warmed to $30^{\circ} \mathrm{C}$. As the color of the mixture turns from yellow-green to yellow-brown, and the color of the slides become green, the slides are removed from the mixture, washed with water, and sonicated for a few 
seconds at room temperature. SiFs-deposited slides were then rinsed with deionized water several times and dried under a stream of nitrogen gas.

\section{S1.2.2. Preparation of the $\beta$-globin mRNA substrate}

The complete protein coding sequence of rabbit $\beta$-globin mRNA was amplified from plasmid pC7ßG [1] by polymerase chain reaction using Pfu DNA polymerase (Stratagene, La Jolla, CA) from primers 5'-GCAGTCTAGAATGGTGCATCTGTCCAG-3' and 5'- GCAC $\underline{\text { AAG }}$ CTTCAGTGGtATtTGTGAGCCAGG-3' (Integrated DNA Technologies, Coralville, IA). Underlined bases indicate the $\mathrm{XbaI}$ and HindIII restriction sites incorporated into the 5'- and 3'termini of the PCR product. This DNA fragment was then inserted into the XbaI + HindIII restriction sites of pGEM7Zf(+) (Promega, Madison, WI) using standard subcloning techniques [2] to generate plasmid pG7(+)ßG-CDS. The fidelity of the $\beta$-globin cDNA insert was verified by restriction digests and automated DNA sequencing.

A 484-nt RNA substrate containing the $\beta$-globin coding sequence (See Figure S1) was prepared by in vitro transcription using T7 RNA polymerase (Ambion, Austin, TX) from a HindIII-linearized pG7(+)ßG-CDS DNA template. Following digestion of template DNA with RQ1-DNase (Promega), templates were purified by duplicate extractions with phenol:chloroform:isoamyl alcohol (25:24:1). Unincorporated nucleotides were removed from the preparation by spin column chromatography through RNase-free G-50 Quick Spin columns (Roche, Indianapolis, IN). The integrity of the $\beta$-globin RNA substrate was evaluated by electrophoresis through formaldehyde-agarose gels stained with ethidium bromide. Fluorescence intensity of ethidium bromide-stained RNA was measured using the EDAS 290 gel documentation system (Kodak, Rochester, NY), with synthesis yield calculated by comparison to co-fractionated RNA size markers (InVitrogen, Carlsbad, CA). 


\section{S1.2.3. MEF-based RNA sensing assays}

The following RNA capture assay [3] was used to detect specific RNA substrates on SiFs-coated glass slides: First, $\beta$-globin mRNA or yeast tRNA substrates (10 ng) were incubated with an antisense primer 5'-GTGAGCCAGGGCATT-TAMRA-3' (fluorescent probe; 10 pmol) in a total volume of $100 \mu$ hybridization buffer [10 mM HEPES·KOH [pH 7.4] containing 100 $\mathrm{mM} \mathrm{KCl}, 2 \mathrm{mM}$ dithiolthreitol, and $1 \mathrm{mM} \mathrm{MgCl}_{2}$ ] at $70{ }^{\circ} \mathrm{C}$ for 5 minutes, then the RNA/DNA construct was slowly cooled to $37^{\circ} \mathrm{C}$ over $20-30$ minutes. The anchor probe (5'-thiolCACCTTCTGATAGGC-3', 10 pmol) was attached to the SiFs by an overnight incubation at $4^{\circ} \mathrm{C}$ in a humidified chamber. Excess thiol-conjugated oligo was removed by washing the surface with the hybridization buffer several times. The TAMRA-linked oligo annealed to RNA substrates were annealed to the thiol-linked anchor oligo on the surface of the SiFs at $37^{\circ} \mathrm{C}$ for 30 minutes in a humidified chamber. Non-binding RNA substrates were removed as described above prior to fluorescence measurements.

Fluorescence measurements on $\mathrm{SiFs}$ were performed by placing the $\mathrm{SiFs}$ on a stationary stage equipped with the fiber-optics mount on a 15-cm-long arm (normal to sample). The output of the fiber was connected to an Ocean Optics HD2000 spectrofluorometer for the emission spectra. The excitation light was provided by a $532 \mathrm{~nm}$ laser at an angle of 45 degrees. The emission spectra were observed through a 532-nm-notch filter (Samrock). 


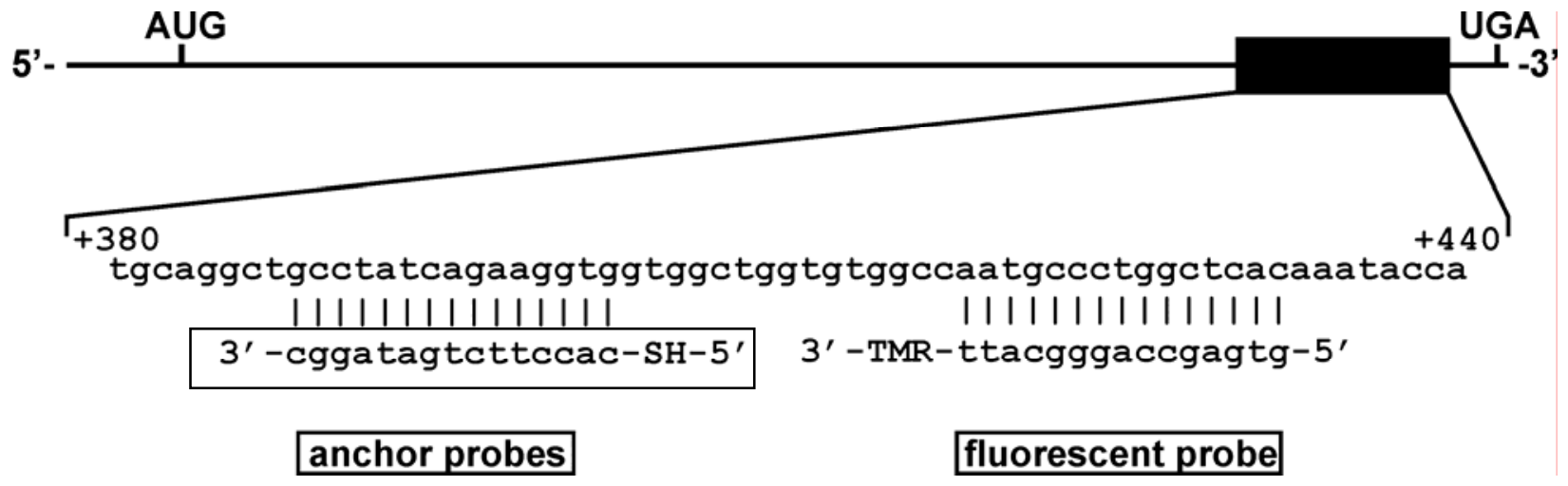

Supporting Information: Figure S1. A schematic of the $\beta$-globin mRNA substrate is shown with the positions of translational initiation (AUG) and termination (UGA) codons indicated. The 3'-coding sequences targeted by the anchor and fluorescent primers are indicated below. Base numbering is relative to the translation initiation codon Accession number for the rabbit bglobin mRNA sequence is V00879.

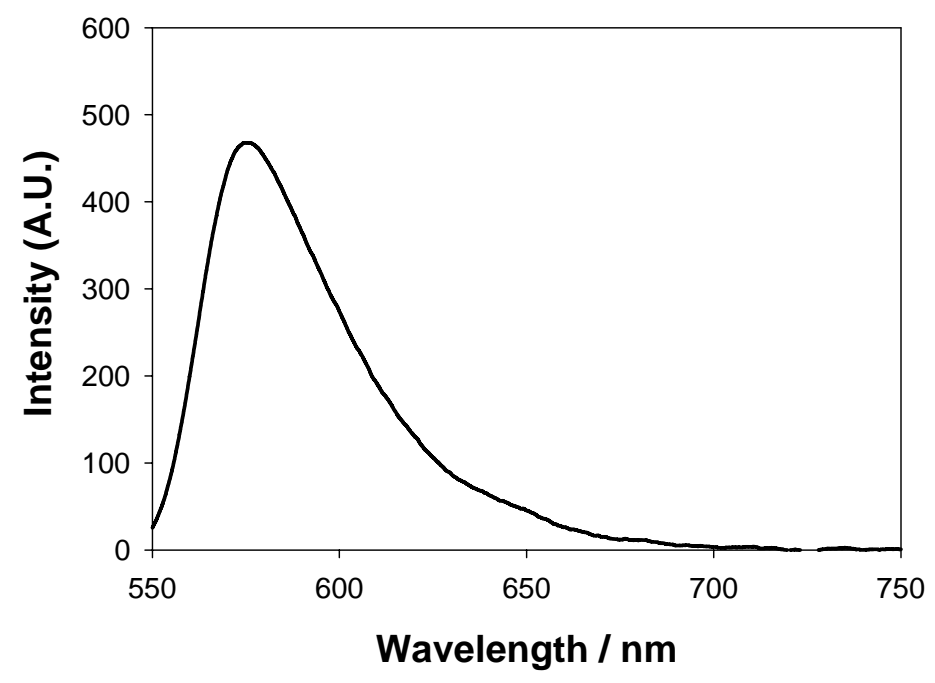

Supporting Information: Figure S2. Fluorescence emission spectrum measured from a $40 \mu \mathrm{L}$ solution of 500 fmoles of TAMRA-linked oligo anchor probe on glass slide (TAMRA-linked oligo is not linked to the surface). 


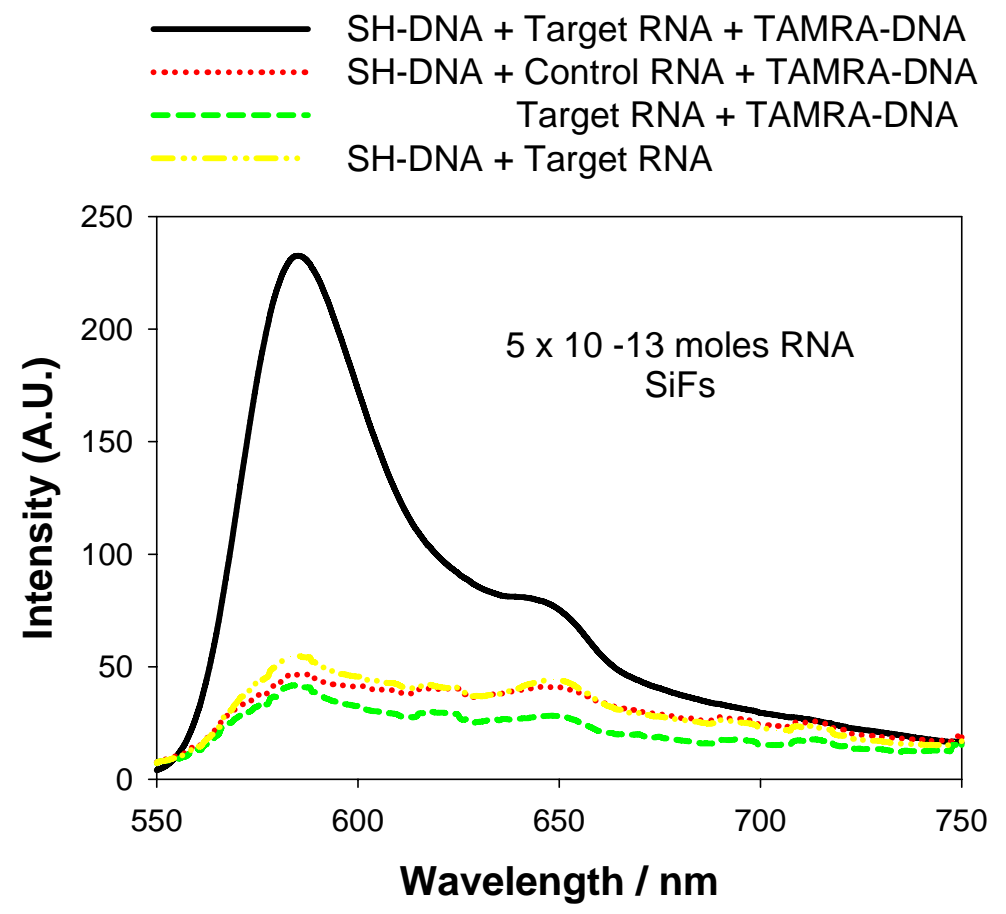

Supporting Information: Figure S3. Fluorescence emission spectra (intensity: arbitrary units) of TAMRA-linked oligo annealed to the 500 fmoles of RNA substrate that was hybridized with the thiolated oligo anchor probe on the surface of the SiFs and control experiments: 1) Control RNA (tRNA, random sequence, Sigma) is used instead of Target RNA, 2) thiolated-oligo anchor probe is omitted, 3) TAMRA-linked oligo is omitted from the RNA capture assay. 

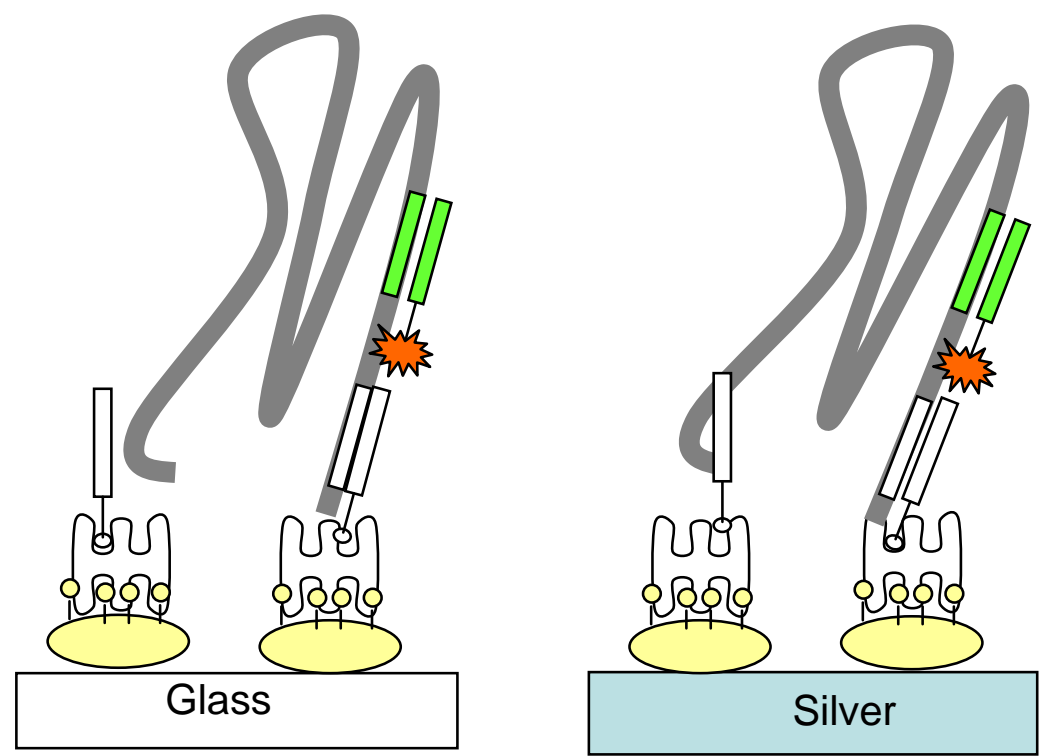

Un: Avidin Biotinylated BSA

$\square$ : Biotinylated 15-mer Oligo

$\sum_{w^{M}}^{M} \square:$ TAMRA-Labeled 15-mer Oligo

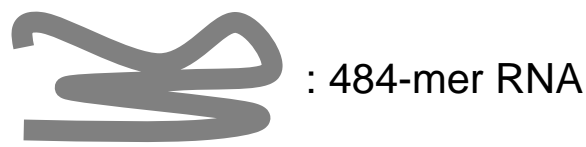

Supporting Information: Figure S4. The experimental scheme used for the detection of RNA in the absence of SiFs (on glass, Top-Left) and in the presence of SiFs using avidin-biotin interactions. In this regard, firstly, the RNA is annealed to a TAMRA-labeled oligo and then the RNA/TAMRA-labeled oligo is annealed to a biotinylated Oligo. Finally, the resultant RNA/Oligo construct is brought the surface due to the interaction of avidin and biotin. 


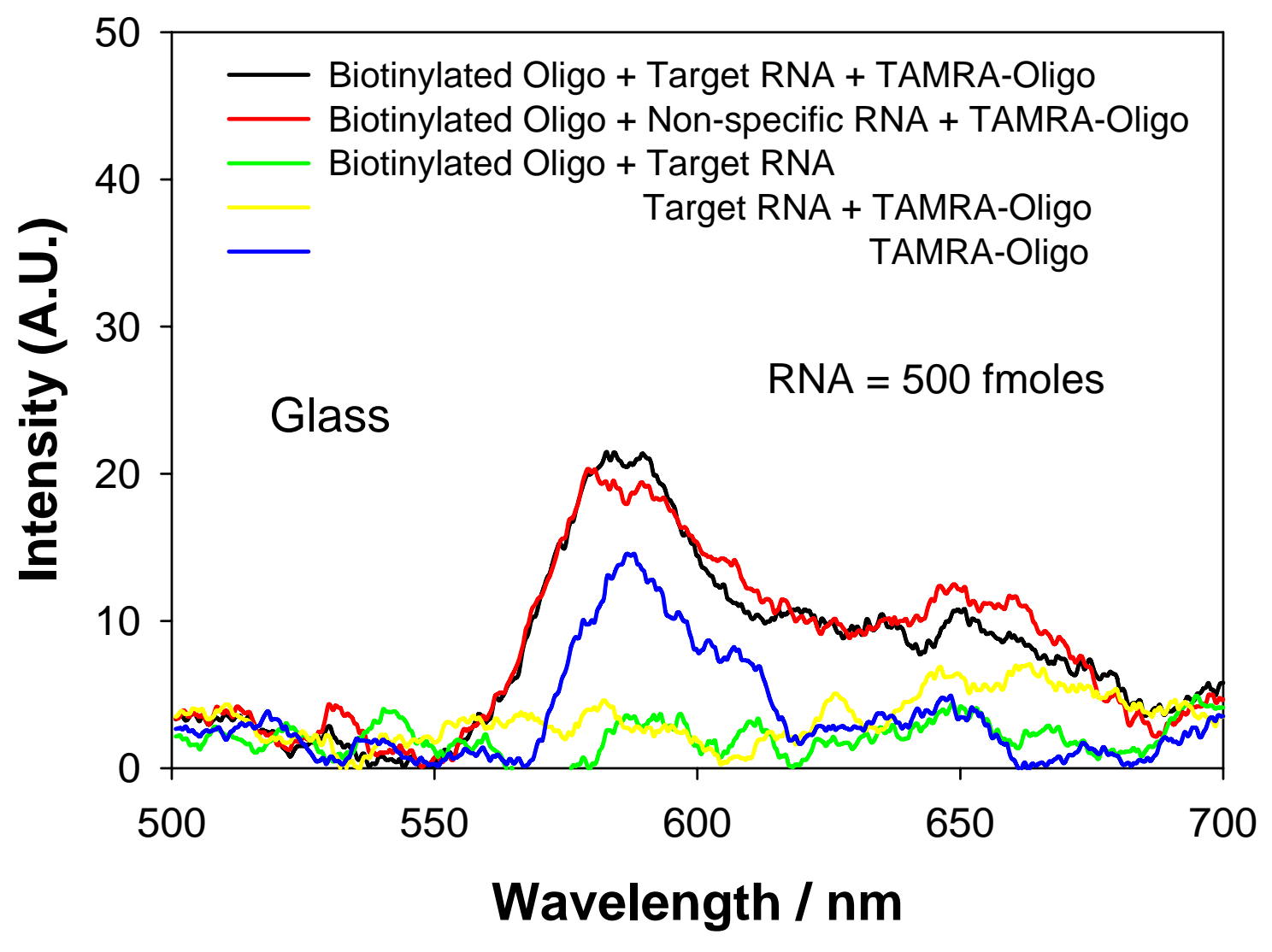

Supporting Information: Figure S5. Fluorescence emission spectra (intensity: arbitrary units) of TAMRA-linked Oligo annealed to the RNA substrate (500 fmoles) that was hybridized with the biotinylated Oligo anchor probe that was brought to the glass surface via avidin-biotin interactions. The emission intensity peak of TAMRA-labeled oligo that was annealed to RNA substrates (Target and non-specific RNA: 500 fmoles) is observed at $585 \mathrm{~nm}$ but are similar (20 $\mathrm{AU})$. That is, the RNA assay on glass substrate in the absence of SiFs is not sensitive enough to distinguish between the actual assay and the non-specific interactions. 


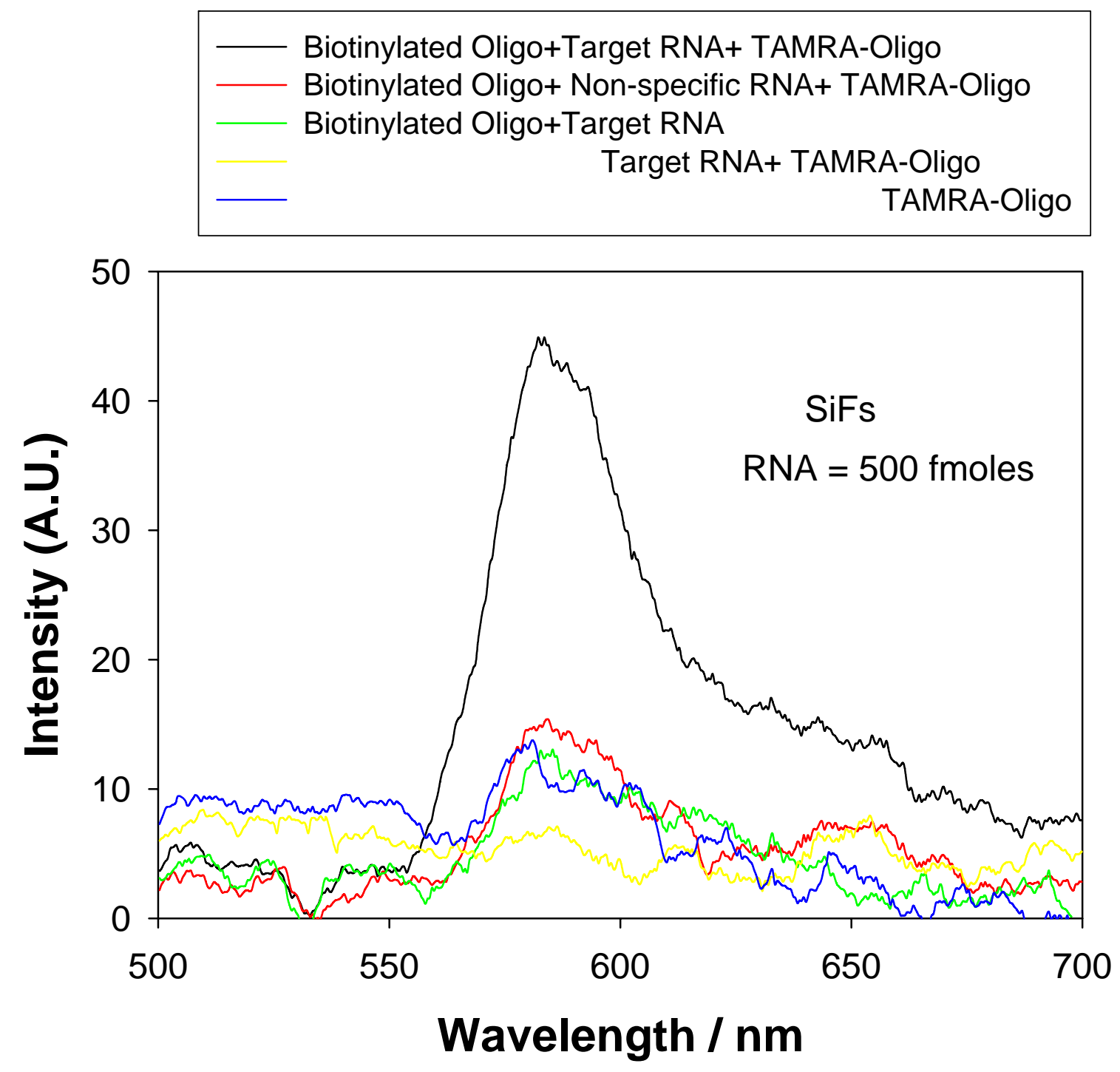

Supporting Information: Figure S6. Fluorescence emission spectra (intensity: arbitrary units) of TAMRA-linked Oligo annealed to the RNA substrate (500 fmoles) that was hybridized with the biotinylated Oligo anchor probe that was brought to the SiFs-coated surface via avidin-biotin interactions. The emission intensity peak of TAMRA-labeled oligo that was annealed to RNA substrates (Target and non-specific RNA: 500 fmoles) is observed at $585 \mathrm{~nm}$ and is significantly 
larger than the background. Although, the sensitivity of the RNA assay using SiFs (and avidinbiotin interactions) is improved compared to the assay on the glass surface, the sensitivity is much less when compared to the RNA assay on SiFs when the thiolated-oligo is used. This is due to the fact that the fluorophore is located approximately $10 \mathrm{~nm}$ away from the surface of the silver when avidin-biotin system is used (the thicknesses of avidin and biotinylated BSA are 4 $\mathrm{nm}$ ) and approximately $4 \mathrm{~nm}$ when thiolated oligo is used. In all the previously published MEF papers (references 13,14,18, 19 in the main text), the maximum enhancement of fluorescence by silver was observed when the fluorophore was located within $8 \mathrm{~nm}$ of the surface and the enhanecement is decreased for the distances larger than $8 \mathrm{~nm}$. Thus, the RNA assay using the thiolated-oligo on SiFs is more sensitive than the assays using avidin-biotin interactions on glass and $\mathrm{SiFs}$.

\section{Reference List for the Supporting Information:}

1. Wilson, G.M., and Deeley, R.G. (1995) An episomal expression vector system for monitoring sequence-specific effects on mRNA stability in human cell lines. Plasmid 33:198-207.

2. Sambrook, J., Fritsch, E.F., and Maniatis, T (1989) Molecular Cloning: A Laboratory Manual, $2^{\text {nd }}$ ed. Cold Spring Harbor: Cold Spring Harbor Laboratory, NY.

3. Tsai, S.P., Wong, A., Mai, E., Chan, P., Mausisa, G., Vasser, M., Jhurani, P., Jakobsen, M.H., Wong, W.L.T., and Stephan, J.-P. (2003) Nucleic acid capture assay, a new method for direct quantitation of nucleic acids. Nucleic Acids Res. 31:e25. 